\title{
NCAM140 and pCREB Expression after Tianeptine Treatment of SH-SY5Y Cells
}

\author{
Mi-gyung Lee ${ }^{1,2 *}$, Daeyoung $\mathrm{Oh}^{3 * \dagger}$, Mi-Ran $\mathrm{Choi}^{4}$, Young-Gyu Chai ${ }^{4}$, \\ Seok-Hyeon $\mathrm{Kim}^{5}$, Dong-Hoon $\mathrm{Oh}^{5}$, and Joonho $\mathrm{Choi}^{3,5}$ 凶 \\ ${ }^{1}$ Department of General Psychiatry, Seoul National Hospital, Seoul, Republic of Korea \\ ${ }^{2}$ Cognitive Neuroscience and Schizophrenia Program, Nathan Kline Institute, Orangeburg, New York, USA \\ ${ }^{3}$ Department of Neuropsychiatry, College of Medicine, Hanyang University, Hanyang University Guri Hospital, Guri, Republic of Korea \\ ${ }^{4}$ Division of Molecular and Life Sciences, Hanyang University, Ansan, Republic of Korea \\ ${ }^{5}$ Department of Neuropsychiatry and Mental Health Institute, College of Medicine, Hanyang University, Seoul, Republic of Korea
}

Objective Antidepressants modulate neuronal plasticity. Tianeptine, an atypical antidepressant, might be involved in the restoration of neuronal plasticity; it primarily enhances the synaptic reuptake of serotonin. NCAM140 is involved in neuronal development processes, synaptogenesis and synaptic plasticity. We investigated the effect of tianeptine on the expression of NCAM140 and its downstream signaling molecule in the human neuroblastoma cell line SH-SY5Y.

Methods NCAM protein expression was measured in human neuroblastoma SH-SY5Y cells that were cultivated in serum-free media and treated with 0,10 , or $20 \mu \mathrm{M}$ tianeptine for 6,24 , or 72 hours. NCAM140 expression in the tianeptine treatment group was confirmed by Western blot, and quantified through measurement of band intensity by absorbance. CREB and pCREB expression was identified after treatment with $20 \mu \mathrm{M}$ tianeptine for 6, 24, and 72 hours by Western blot.

Results Compared to cells treated for 6 hours, cells treated with 0 or $10 \mu \mathrm{M}$ tianeptine for 72 hours showed a significant increase in NCAM140 expression and cells treated with $20 \mu \mathrm{M}$ tianeptine showed a significant increase after 24 and 72 hours. The pCREB level in cells treated with $20 \mu \mathrm{M}$ tianeptine increased in time-dependent manner.

Conclusion Our findings indicated that the tianeptine antidepressant effect may occur by induction of NCAM140 expression and CREB phosphorylation.

Psychiatry Investig 2014;11(3):313-318

Key Words Tianeptine, NCAM140, CREB, Antidepressant, Neuroblastoma cells.

\section{INTRODUCTION}

Mood disorders are among the most prevalent forms of mental illness. Depression is a widespread and heterogeneous disorder with severe health and socioeconomic consequences. ${ }^{1}$

Received: June 5, 2013 Revised: October 4, 2013

Accepted: October 23, 2013 Available online: July 21, 2014

$\triangle$ Correspondence: Joonho Choi, MD

Department of Neuropsychiatry, Hanyang University Guri Hospital, 153

Gyeongchun-ro, Guri 471-701, Republic of Korea

Tel: +82-31-560-2272, Fax: +82-31-554-2599

E-mail: jchoi@hanyang.ac.kr

*These authors contributed equally to the work.

${ }^{\dagger}$ Current Affiliation

Department of Psychiatry, CHA Bundang Medical Center, CHA University, Seongnam, Republic of Korea

@ This is an Open Access article distributed under the terms of the Creative Commons Attribution Non-Commercial License (http://creativecommons.org/licenses/bync/3.0) which permits unrestricted non-commercial use, distribution, and reproduction in any medium, provided the original work is properly cited.
The monoamine hypothesis of depression, which includes dysfunction in the serotonergic, noradrenergic and possibly dopaminergic systems, is widely accepted as the pathophysiology of depression. The introduction of selective serotonin reuptake inhibitors (SSRIs) and serotonin-norepinephrine reuptake inhibitors (SNRIs) gave clinicians a safer medication choice than tricyclic antidepressants (TCAs). ${ }^{2}$ However, the efficacy of SSRIs and SNRIs is not better than that of TCAs, suggesting the need for rational drug discovery of antidepressants. Tianeptine is a newer antidepressant with unique neurochemical properties that are different from other antidepressants. ${ }^{3}$ In contrast to TCAs and SSRIs, tianeptine is suggested to decrease serotoninergic activity and the amount of serotonin in serotonergic synapses by increasing serotonin reuptake; it is hence called a serotonin reuptake enhancer. ${ }^{4-6}$ Clinically, tianeptine has an efficacy equivalent to the classic antidepressants. ${ }^{7}$

Tianeptine decreases the axonal atrophy of neurons that is 
precipitated by stress and prevents the loss of spatial memory induced by chronic stress and the cognitive deterioration that results from drinking alcohol. ${ }^{8,9}$ Shortening of dendritic length in the pyramidal neurons of the CA3 region of rats exposed to stress was observed only in a group treated with tianeptine, and not in a group treated with fluoxetine..$^{10}$

Depression is considered to be caused by structural changes in molecules such as monoamines in the brain, and antidepressants function by counteracting these molecular changes. However, several observations suggest an alternative hypothesis to this view of depression. ${ }^{11}$ According to the network hypothesis, depression results from an abnormality of information processing within neural networks, and antidepressant drugs improve the plasticity of neuronal connectivity. ${ }^{12}$ Experimental studies of stress and antidepressant treatment implicated neurogenesis in the etiology of depression. Antidepressant drugs increase the regeneration and turnover of neural cells to replace apoptotic cells with new viable cells. Antidepressants also induce neural plasticity by contributing to the maturation of new neural cells. ${ }^{11,13}$

In general, the improvement of depressive symptoms by long-term antidepressant administration is attributed to the restoration of neural plasticity. ${ }^{14}$ One of the indexes of the restoration of neural plasticity is neural cell adhesion molecule (NCAM). NCAM is a cell-surface protein that contains five Iglike and two fibronectin type III repeats followed by a variably spliced cytoplasmic domain that produces two major transmembrane isoforms (180 and $140 \mathrm{kDa}$ ) and a glycophosphatidyl inositol-linked isoform (120 kDa). The cytoplasmic domains of the NCAM isoforms link to the actin cytoskeleton by directly binding to spectrin and also interact with integrin to activate signaling pathways. ${ }^{15}$ The homophilic interaction of NCAM promotes the binding of Fyn (a nonreceptor tyrosine kinase) and Fak (a focal adhesion kinase) and their phosphorylation, which activates the Ras-mitogen-activated protein kinase (MAPK) pathway. The cyclic-AMP response element binding protein (CREB) is subsequently phosphorylated, resulting in neurite outgrowth. Consequently, NCAM promotes survival and differentiation of neurons, axon growth, and synapse formation..$^{16,17}$

Among the three NCAM isoforms, the expression level of NCAM140 is sensitively regulated by chronic stress. Downregulation of NCAM140 is implicated in the structural alterations of the hippocampus induced by chronic stress exposure. ${ }^{18}$ In addition, NCAM140 has protective activity against oxidative stress. ${ }^{19}$

NCAM140 is known to be important in the formation and maintenance of the neural network. However, little is known about the role of NCAM140 in antidepressant action. Previous studies reported that fluoxetine affects NCAM140 expres- sion. ${ }^{20,21}$ The aim of this study was to identify the effect of tianeptine treatment on expression of NCAM140 and downstream signaling molecules. To explore this, we measured the expression of NCAM140 and CREB/phosphor-CREB (pCREB) in SH-SY5Y cells after tianeptine treatment. We identified significant upregulation of NCAM expression and phosphorylation of CREB in tianeptine-treated SH-SY5Y cells. These results suggested that the antidepressant effect of tianeptine might result from the regulation of NCAM expression and CREB phosphorylation.

\section{METHODS}

\section{Cell culture}

SH-SY5Y, a human neuroblastoma cell line, was cultured in Dulbecco's modified Eagle's medium (DMEM) (Gibco BRL) containing 10\% FBS (Gibco BRL). DMEM culture solution was changed every 48 hours until cell maturation. For experimental treatment, tianeptine (Sigma-Aldrich) was prepared at 10 or $20 \mu \mathrm{M}$ before addition to cell cultures.

\section{Protein separation and measurement}

Protein separation was performed using the iNtRON Biotechnology kit. First, 10 or $20 \mu \mathrm{M}$ tianeptine was added to SHSY5Y cells. After 6, 24, or 72 hours of culture, cells were washed with PBS and harvested by 2000-3000 rpm centrifugation. To $5 \times 10^{6} \mathrm{SH}-\mathrm{SY} 5 \mathrm{Y}$ cells, $400 \mu \mathrm{L}$ Pro-Prep was added with mixing and cells were incubated on ice for 20 minutes. After 13,000 rpm centrifugation for 5 minutes at $4^{\circ} \mathrm{C}$, proteins in the upper layer were collected and stored at $-20^{\circ} \mathrm{C}$ for Western blotting and quantitative analysis. Protein assay kit reagents (Bio-Rad) were diluted 1:5 with distilled water, and $1,0.5,0.1,0.01$, or 0 $\mathrm{mg} / \mathrm{mL}$ BSA standards were set up. After placing $10 \mu \mathrm{L}$ of BSA standards in 96-well plates, $200 \mu \mathrm{L}$ working solution was added. Prepared BSA standards and protein samples to be measured were placed at room temperature for 5 minutes. Absorbance was measured with an ELISA reader at $595 \mathrm{~nm}$, and protein concentration was measured using the standard curve.

\section{Western blot analysis}

To observe NCAM140, CREB and pCREB, Western blots were performed with protein extracted from cells using polyclonal rabbit anti-NCAM $(1: 10,000)$ (Chemicon), anti-CREB or anti-pCREB $(1: 1,000)$ (Cell Signaling Technology). Protein samples were boiled for 5 minutes in Laemmli sample solution (62.5 mM Tris-Cl pH 6.8, 2\% SDS, 10\% glycol, $0.5 \%$ $\beta$-mercaptoethanol, $10 \mu \mathrm{g} / \mathrm{ml}$ bromophenol blue), and $80 \mathrm{~V}$ electrophoresis was carried out using $8 \%$ or $12 \%$ polyacrylamide-gels, and proteins in $20 \%$ methanol were electrotransferred at $30 \mathrm{~V}$ at $4^{\circ} \mathrm{C}$ overnight to nitrocellulose membranes. 
Membranes were incubated in TBST buffer (20 mM Tris-HCL pH 7.6, $500 \mathrm{mM} \mathrm{NaCl}, 0.1 \%$ Tween 20) containing 5\% nonfat dry milk and stirred at room temperature for 1 hour. Respective antibodies were added with stirring at $4^{\circ} \mathrm{C}$ in TBST buffer overnight. Membranes were washed with TBST buffer 3 times for 10 minutes each, then washed with TBST buffer containing 1:2000 HRP-conjugated anti-rabbit IgG. ECL+reagents (Amersham Bioscience) were added at 1:40 and membranes were exposed to film. Experiments were repeated 6 times for data validation.

\section{Statistics}

One-way analysis of variance was performed using SPSS version 12.0 (SPSS Inc., Chicago, IL, USA), with post hoc analysis using Tukey's honestly significant difference (HSD) test.

\section{RESULTS}

In a previous study, administration of $10 \mu \mathrm{M}$ tianeptine had a neuroprotective effect on damaged neuronal cells. ${ }^{22}$ Hence, we used $10 \mu \mathrm{M}$ tianeptine as an appropriate effective dose and $20 \mu \mathrm{M}$ as a higher effective dose. To evaluate the viability of SH-SY5Y cells after tianeptine treatment, we incubated SHSY5Y cells in media with 0,10 or $20 \mu \mathrm{M}$ tianeptine and maintained cells for up to 3 days. The proliferation and morphology of SH-SY5Y cells was not affected by tianeptine (data not shown). We therefore treated cells with 0,10 or $20 \mu \mathrm{M}$ tianeptine for 72 hours.

\section{Dosage effect of tianeptine on NCAM140 expression}

We explored the possibility that the increasing doses of tianeptine affected NCAM140 expression in SH-SY5Y cells. Tianeptine was administered to SH-SY5Y cells for 6, 24, or 72 hours at 0,10 , or $20 \mu \mathrm{M}$. The effects of tianeptine treatment on NCAM expression in SH-SY5Y cells were measured using immunoblotting (Figure 1). In $0 \mu \mathrm{M}$ tianeptine-treated cells, NCAM140 expression increased by more than 105\% at 24 hours and significantly increased by almost $143 \%$ at 72 hours compared to cells treated with tianeptine for 6 hours (Figure 1A). In $10 \mu \mathrm{M}$ tianeptine-treated cells, NCAM140 expression increased by more than $112 \%$ at 24 hours and significantly increased by $254 \%$ at 72 hours. NCAM140 expression significantly increased by more than $190 \%$ at 24 hours and by $439 \%$ at 72 hours in $20 \mu \mathrm{M}$ tianeptine-treated cells compared to at 6 hours. Moreover, NCAM140 expression after $20 \mu \mathrm{M}$ tianeptine treatment increased earlier and to higher levels than after $10 \mu \mathrm{M}$ tianeptine treatment. These results suggested that the tianeptine effect on expression of NCAM140 might be dependent on dose and length of administration.

To explore the expression levels of NCAM140 at 72 hours, we administered 0,10 , or $20 \mu \mathrm{M}$ tianeptine to SH-SY5Y cells and performed immunoblotting (Figure 2). We found that 20 $\mu \mathrm{M}$ tianeptine treatment resulted significantly increased NCAM140 expression compared to $0 \mu \mathrm{M}$ treatment. In cells treated with $10 \mu \mathrm{M}$ tianeptine, the expression level of NCAM140 was not significantly different than cells treated with $0 \mu \mathrm{M}$ tianeptine but expression showed a tendency to increase. These results suggested that administration of tianeptine induced expression of NCAM140.
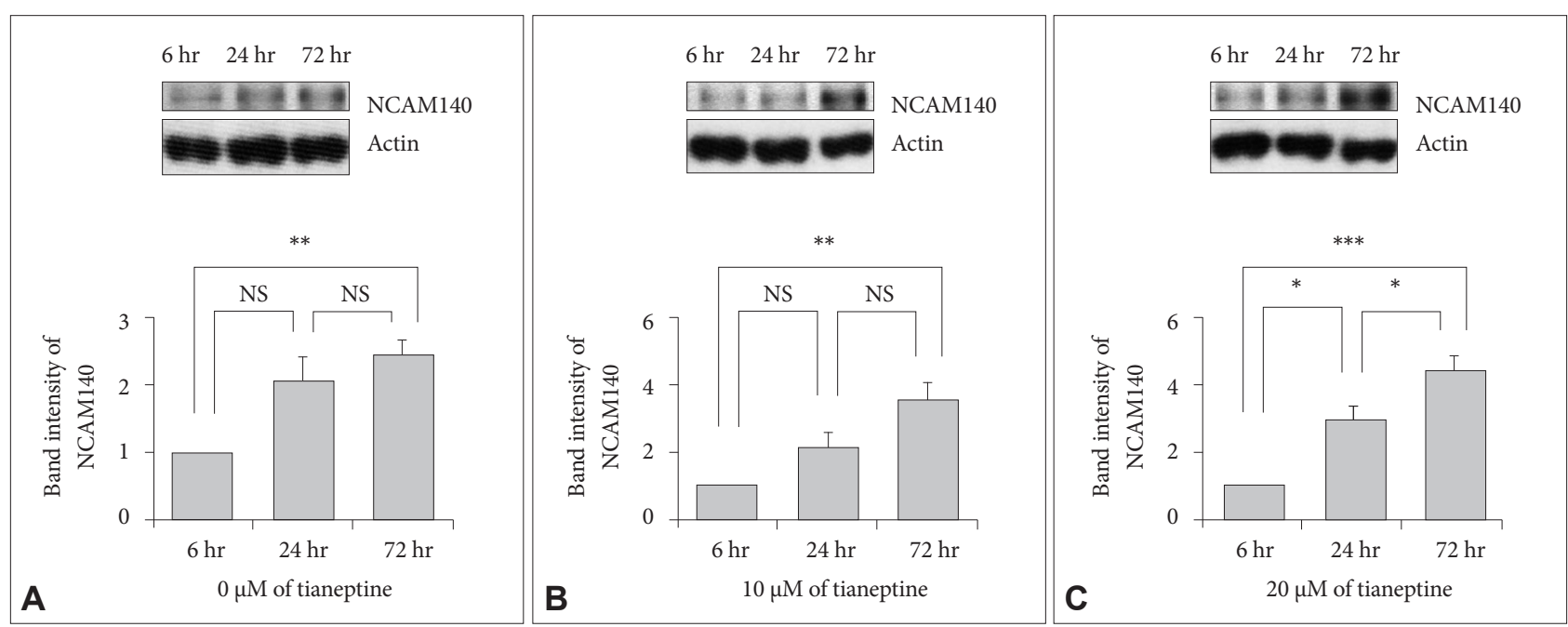

Figure 1. Effect of tianeptine on NCAM140 expression in SH-SY5Y cells. Cells were treated with various concentrations of tianeptine for 6 , 24 or 72 hours. NCAM140 protein was measured by Western blot, with actin as an internal control. Tianeptine was administered to SHSY5Y cells at $0 \mu \mathrm{M}(\mathrm{A}), 10 \mu \mathrm{M}(\mathrm{B})$, or $20 \mu \mathrm{M}(\mathrm{C})$. NCAM140 intensity values are mean \pm SEM $(n=6)$ of the fold-change from cells cultured for 6 hours. Significant differences from 6 hours by one-way ANOVA, followed by Tukey's HSD post hoc test. ${ }^{*} p<0.05,{ }^{* *} p<0.01,{ }^{* * *} p<0.001$. NS: not significant, NCAM: neural cell adhesion molecule. 


\section{Effect of tianeptine on CREB expression and phosphorylation}

Previously, fluoxetine treatment was found to activate NCAM140 expression and CREB phosphorylation in rat C6 glioma cells. ${ }^{21}$ We explored whether tianeptine treatment affected expression and phosphorylation of CREB. SH-SY5Y cells were treated with $20 \mu \mathrm{M}$ tianeptine for 6,24 , or 72 hours and CREB and pCREB expression was measured by immunoblot (Figure 3). The CREB expression level at 24 or 72 hours was similar to the level at 6 hours. However, CREB phosphorylation gradually increased with time. These results indicated that tianeptine treatment seemed to activate CREB phosphorylation without affecting the total CREB level.

\section{DISCUSSION}

In this study, we found that cells treated with tianeptine for 72 hours showed a significant increase in NCAM140 expression compared to cells treated for 6 hours. In particular, 20 $\mu \mathrm{M}$ tianeptine for 24 hours induced a significant increase in NCAM140 expression in contrast with 0 or $10 \mu \mathrm{M}$ tianeptine treatment (Figure 1). NCAM140 expression seemed to have a dose-dependent effect because $20 \mu \mathrm{M}$ tianeptine for 72 hours significantly increased NCAM140 expression compared to 0 $\mu \mathrm{M}$ tianeptine and $10 \mu \mathrm{M}$ tianeptine tended to modestly increase NCAM140 expression compared to $0 \mu \mathrm{M}$ tianeptine (Figure 2).

Chronic treatment with imipramine, a tricyclic antidepressant, is reported to induce an increase in PSA-NCAM in the rat prelimbic cortex. ${ }^{23}$ Fluoxetine treatment of rat $\mathrm{C} 6$ glioma cells increases NCAM140 expression in a time-dependent manner. ${ }^{21}$ In contrast, a previous study reported that venlafaxine, an SNRI, attenuated the NCAM increase in the hilus area of the mouse hippocampus. ${ }^{24}$ Thus, the regulation of NCAM140 expression might be important in depression treatment.

Previous studies on the antidepressant effect and action mechanism of TCAs and SSRIs demonstrated that longer treatment of antidepressants is more important than any other condition of treatment. ${ }^{25}$ Some studies compared antidepressant and non-antidepressant treatment in rodents exposed to repeated chronic stress. ${ }^{26,27}$ Other studies measured the level of molecular markers representative of synaptic plasticity with diverse antidepressants treatment. ${ }^{28,29}$ The findings of these studies demonstrated that chronic stress exposure, which can precipitate or exacerbate depression, disrupts neuroplasticity. Antidepressant treatment has an opposing effect and can enhance neuroplasticity including structural plasticity changes in dendritic morphology and adult neurogenesis, and physiological synaptic plasticity. Chronic administration of a TCA blocked the decrease in spine density in the CA3 and CA1 cell layers of dentate gyrus in the hippocampus. ${ }^{30}$ Interestingly, chronic stress decreases the number and length of apical dendrites of CA3 pyramidal cells in the hippocampus. ${ }^{31}$ This reduction in dendritic length and complexity is blocked or reversed by chronic administration of tianeptine, but not by administration of a fluoxetine. ${ }^{10}$ Our data suggested that treatment of depressed patients with tianeptine might modulate the neural network by upregulating NCAM140 expression and resulting in improved mood.

Previous data demonstrates that NCAM induces recruitment of the tyrosine kinase Fyn to the NCAM intracellular domain, which in turn activates Fak, ERK, and the transcription factor

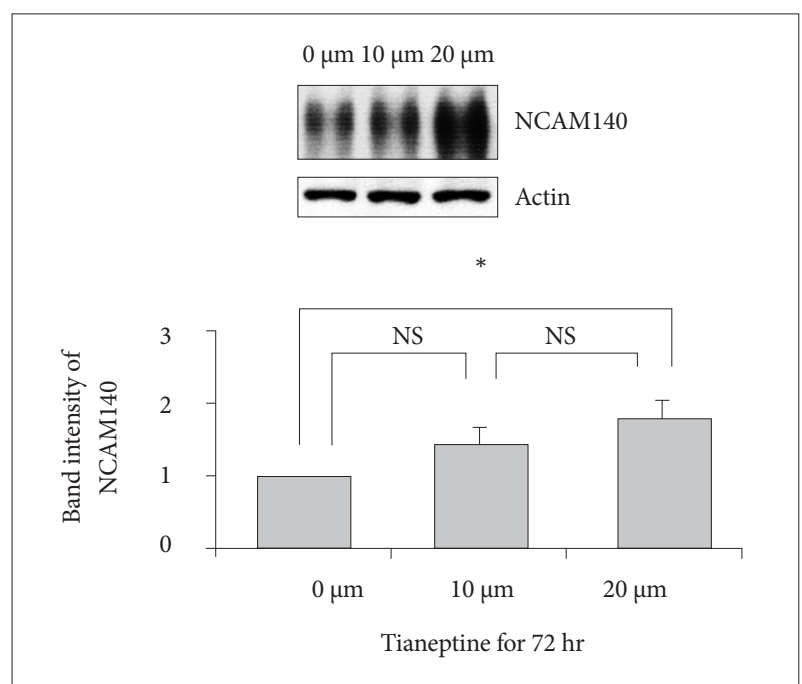

Figure 2. Effect of tianeptine for 72 hours on NCAM140 expression in SH-SY5Y cells. Cells were treated with 0,10 , or $20 \mu \mathrm{M}$ tianeptine for 72 hours. NCAM140 protein was measured by western blot, with actin as an internal control. NCAM140 intensity values are mean \pm SEM $(n=6)$ of fold-change from cells treated with $0 \mu \mathrm{M}$ tianeptine. Significant differences from 6 hours by oneway ANOVA, followed by Tukey's HSD post hoc test. " $p<0.05$. NS: not significant.

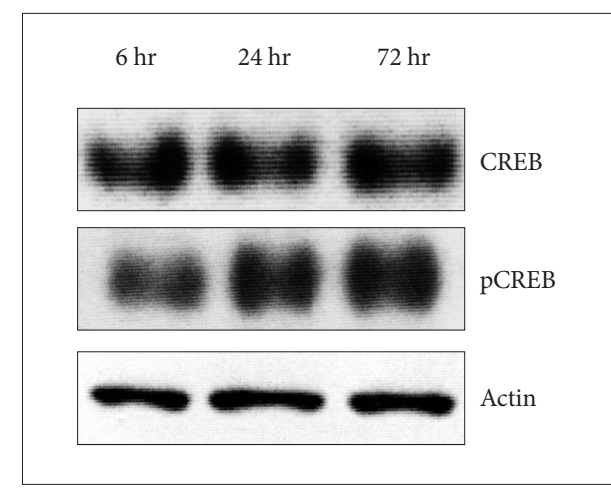

Figure 3. Effect of tianeptine on CREB and pCREB expression in SH-SY5Y cells. Cells were treated with $20 \mu \mathrm{M}$ tianeptine for 6 , 24 , or 72 hours. CREB and PCREB protein was measured by Western blot, with actin as an internal control. CREB: cyclic-AMP response element binding protein, $\mathrm{pCREB}$ : phosphorylated form of CREB. 
CREB, which stimulates neurite outgrowth. ${ }^{32}$ CREB transcriptional activity depends on its phosphorylation state, with phosphorylation resulting in the activation of cAMP response element-mediated gene transcription and neuronal remodeling such as neurite outgrowth. ${ }^{33}$ In this study, CREB phosphorylation increased time-dependently with $20 \mu \mathrm{M}$ tianeptine treatment (Figure 3). Future studies are needed to determine whether tianeptine treatment has an impact on modulating neuronal plasticity and neurite outgrowth by phosphorylating and activating CREB via NCAM140-induced activation of the Ras-MAPK pathway.

Increasing evidence suggests that the glutamatergic system, which is known to play a major role in neuronal plasticity and cellular resilience, may be involved in the pathophysiology and treatment of depression. ${ }^{34}$ In addition, the network hypothesis states that depression is defined as a disorder with impairment in information processing with structural changes in the brain. ${ }^{12}$ In this hypothesis, increased glutamate induced by stress might result in cellular excitotoxicity in the brain. ${ }^{35}$ Tianeptine treatment normalizes impaired glutamatergic neurotransmission in the hippocampus and amygdala induced by stress. Furthermore, tianeptine modulates excitatory synaptic currents of the hippocampal CA3 synapses to regulate the phosphorylation state of glutamate receptors in chronically stressed rodents ${ }^{36}$ and increases basal transmission of glutamatergic excitatory synapse. ${ }^{37}$ Finally, facilitation of AMPA receptor-mediated transmission leads to activation of the NCAM promoter, indicating that that NCAM synthesis is regulated in part by synaptic activity. ${ }^{38}$ Thus, tianeptine might induce NCAM140 expression by modulating glutamatergic transmission.

Our findings must be reviewed in the context of the study's limitations. We were unable to exclude that the antidepressant might regulate other isoforms of NCAM in addition to NCAM140. Experiments were performed only a single time for pCREB immunoblot data, so we were able to determine statistical significance. Additional studies are needed to establish these findings. We were unable to demonstrate the impact of the serotonergic modulating function of tianeptine on expression of NCAM140 and PCREB. We will investigate this mechanism in the future.

According to our findings, tianeptine has a distinct mechanism that is unlike other antidepressants. Therefore, studying the effects of tianeptine in patients with depression who are not adequately treated by SSRIs would be clinically informative. In conclusion, our study suggests that tianeptine has antidepressant effects through increased expression of NCAM140 and phosphorylation of CREB.

\section{Acknowledgments}

This work was supported by the research fund of Hanyang University
(HY-2007).

\section{REFERENCES}

1. Kessler RC, Berglund P, Demler O, Jin R, Koretz D, Merikangas KR, et al. The epidemiology of major depressive disorder: results from the National Comorbidity Survey Replication (NCS-R). JAMA 2003;289: 3095-3105.

2. Stahl SM, Grady MM, Moret C, Briley M. SNRIs: their pharmacology, clinical efficacy, and tolerability in comparison with other classes of antidepressants. CNS Spectr 2005;10:732-747.

3. Uzbay TI. Tianeptine: potential influences on neuroplasticity and novel pharmacological effects. Prog Neuropsychopharmacol Biol Psychiatry 2008;32:915-924.

4. Mennini T, Mocaer E, Garattini S. Tianeptine, a selective enhancer of serotonin uptake in rat brain. Naunyn Schmiedebergs Arch Pharmacol 1987;336:478-482.

5. McEwen BS, Conrad CD, Kuroda Y, Frankfurt M, Magarinos AM, McKittrick C. Prevention of stress-induced morphological and cognitive consequences. Euro Neuropsychopharmacol 1997;7(Suppl 3):S323S328.

6. Conrad CD, Galea LA, Kuroda Y, McEwen BS. Chronic stress impairs rat spatial memory on the Y maze, and this effect is blocked by tianeptine pretreatment. Behav Neurosci 1996;110:1321-1334.

7. Kasper S, Olie JP. A meta-analysis of randomized controlled trials of tianeptine versus SSRI in the short-term treatment of depression. Eur Psychiatry 2002;17(Suppl 3):331-340.

8. Beracochea D, Deslandes A, Jaffard R. Comparison of the effects of tianeptine and paroxetine on amnesic deficits induced by prolonged alcohol drinking in mice. Encephale 1994;20:13-16.

9. Jaffard R, Mocaer E, Lebrun C, Beracochea D. Effects of tianeptine on learning and memory in mice. Improvement of impairments induced by chronic alcoholism and brain aging. Presse Med 1991;20:1812-1816.

10. Magarinos AM, Deslandes A, McEwen BS. Effects of antidepressants and benzodiazepine treatments on the dendritic structure of CA3 pyramidal neurons after chronic stress. Euro J Pharmacol 1999;371:113122.

11. Sairanen M, Lucas G, Ernfors P, Castren M, Castrén E. Brain-derived neurotrophic factor and antidepressant drugs have different but coordinated effects on neuronal turnover, proliferation, and survival in the adult dentate gyrus. J Neurosci 2005;25:1089-1094.

12. Castrén E. Is mood chemistry? Nat Rev Neurosci 2005;6:241-246.

13. Fujioka T, Fujioka A, Duman RS. Activation of cAMP signaling facilitates the morphological maturation of newborn neurons in adult hippocampus. J Neurosci 2004;24:319-328.

14. Santarelli L, Saxe M, Gross C, Surget A, Battaglia F, Dulawa S, et al. Requirement of hippocampal neurogenesis for the behavioral effects of antidepressants. Science 2003;301:805-809.

15. Schmid RS, Maness PF. L1 and NCAM adhesion molecules as signaling coreceptors in neuronal migration and process outgrowth. Curr Opin Neurobiol 2008;18:245-250.

16. Schachner M. Neural recognition molecules and synaptic plasticity. Curr Opin Cell Biol 1997;9:627-634.

17. Kiss JZ, Troncoso E, Djebbara Z, Vutskits L, Muller D. The role of neural cell adhesion molecules in plasticity and repair. Brain Res Brain Res Rev 2001;36:175-184.

18. Touyarot K, Sandi C. Chronic restraint stress induces an isoform-specific regulation on the neural cell adhesion molecule in the hippocampus. Neural Plast 2002;9:147-159.

19. Feng Z, Li L, Ng PY, Porter AG. Neuronal differentiation and protection from nitric oxide-induced apoptosis require c-Jun-dependent expression of NCAM140. Mol Cell Biol 2002;22:5357-5366.

20. Choi MR, Choi MR, Baik SY, Baik SY, Jung KH, Jung KH, et al. Characterization of differentially expressed genes upon chronic fluoxetine treatment in rat C6 glioma cells. Korean J Psychopharmacol 2004;15: 
457-467.

21. Choi MR, Oh DH, Kim SH, Jung KH, Das ND, Chai YG. Fluoxetine increases the expression of NCAM140 and pCREB in rat C6 glioma cells. Psychiatry Investig 2012;9:180-186.

22. Plaisant F, Dommergues MA, Spedding M, Cecchelli R, Brillault J, Kato G, et al. Neuroprotective properties of tianeptine: interactions with cytokines. Neuropharmacology 2003;44:801-809.

23. Sairanen M, O'Leary OF, Knuuttila JE, Castren E. Chronic antidepressant treatment selectively increases expression of plasticity-related proteins in the hippocampus and medial prefrontal cortex of the rat. Neuroscience 2007;144:368-374.

24. Fang S, Yan B, Wang D, Bi X, Zhang Y, He J, et al. Chronic effects of venlafaxine on synaptophysin and neuronal cell adhesion molecule in the hippocampus of cerebral ischemic mice. Biochem Cell Biol 2010; 88:655-663.

25. Sandi C, Bisaz R. A model for the involvement of neural cell adhesion molecules in stress-related mood disorders. Neuroendocrinology 2007; 85:158-176.

26. Cryan JF, Markou A, Lucki I. Assessing antidepressant activity in rodents: recent developments and future needs. Trends Pharmacol Sci 2002;23:238-245.

27. Berton O, Nestler EJ. New approaches to antidepressant drug discovery: beyond monoamines. Nat Rev Neurosci 2006;7:137-151.

28. Pittenger C, Duman RS. Stress, depression, and neuroplasticity: a convergence of mechanisms. Neuropsychopharmacology 2008;33:88-109.

29. Nestler EJ, Barrot M, DiLeone RJ, Eisch AJ, Gold SJ, Monteggia LM. Neurobiology of depression. Neuron 2002;34:13-25.

30. Norrholm SD, Ouimet CC. Altered dendritic spine density in animal models of depression and in response to antidepressant treatment.
Synapse 2001;42:151-163.

31. McEwen BS. Stress and hippocampal plasticity. Ann Rev Neurosci 1999;22:105-122

32. Kolkova K, Novitskaya V, Pedersen N, Berezin V, Bock E. Neural cell adhesion molecule-stimulated neurite outgrowth depends on activation of protein kinase $\mathrm{C}$ and the Ras-mitogen-activated protein kinase pathway. J Neurosci 2000;20:2238-2246.

33. D'Sa C, Duman RS. Antidepressants and neuroplasticity. Bipolar Disord 2002;4:183-194.

34. Zarate CA Jr, Du J, Quiroz J, Gray NA, Denicoff KD, Singh J, et al. Regulation of cellular plasticity cascades in the pathophysiology and treatment of mood disorders: role of the glutamatergic system. Ann N Y Acad Sci 2003;1003:273-291.

35. Mark LP, Prost RW, Ulmer JL, Smith MM, Daniels DL, Strottmann JM, et al. Pictorial review of glutamate excitotoxicity: fundamental concepts for neuroimaging. AJNR Am J Neuroradiol 2001;22:18131824.

36. Kole MHP, Swan L, Fuchs E. The antidepressant tianeptine persistently modulates glutamate receptor currents of the hippocampal CA3 commissural associational synapse in chronically stressed rats. European J Neurosci 2002;16:807-816.

37. Budziszewska B, Jaworska-Feil L, Kajta M, Lasoń W. Antidepressant drugs inhibit glucocorticoid receptor-mediated gene transcription - a possible mechanism. Br J Pharmacol 2000;130:1385-1393.

38. Holst BD, Vanderklish PW, Krushel LA, Zhou W, Langdon RB, McWhirter JR, et al. Allosteric modulation of AMPA-type glutamate receptors increases activity of the promoter for the neural cell adhesion molecule, N-CAM. Proc Natl Acad Sci U S A 1998;95:2597-2602. 